\title{
PUMCH-SLN nomogram for the preoperative prediction of sentinel lymph node metastasis in breast cancer
}

\section{Xue-fei Wang}

Peking Union Medical College and Hospital

Guo-chao Zhang

Chinese Academy of Medical Sciences and Peking Union Medical College

Zhi-chao Zuo

Xiangtan Central Hospital

Qing-li Zhu

Peking Union Medical College Hospital

Zhen-zhen Liu

Peking Union Medical College

\section{Sha-fei Wu}

Peking Union Medical College Hospital

\section{Jia-xin Li}

Peking Union Medical College and Hospital

Jian-hua Du

Peking Union Medical College and Hospital

\section{Cun-li Yan}

Baoji Maternal and Child Health Hospital

\section{Xiao-ying Ma}

Qinghai Provincial People's Hospital

\section{Yue Shi}

Shanxi Traditional Chinese Medical Hospital

He Shi

Shanxi Traditional Chinese Medical Hospital

\section{Yi-dong Zhou}

Peking Union Medical College and Hospital

\section{Feng Mao}

Peking Union Medical College and Hospital

\section{Yan Lin}

Peking Union Medical College and Hospital

\section{Song-jie Shen}


Peking Union Medical College and Hospital

\section{Xiao-hui Zhang}

Peking Union Medical College and Hospital

Qiang Sun ( $\square$ sunqiangpumc2020@sina.com )

Peking Union Medical College Hospital

\section{Research}

Keywords: breast cancer; axillary lymph node (ALN); ultrasound; nomogram

Posted Date: August 3rd, 2020

DOI: https://doi.org/10.21203/rs.3.rs-51231/v1

License: (c) (i) This work is licensed under a Creative Commons Attribution 4.0 International License. Read Full License 


\section{Abstract}

Background: To investigate associations between clinicopathological features and sentinel lymph node (SLN) status of breast cancer, then constructed a nomogram to predict SLN metastasis.

Methods: Patients treated with SLN dissection from 2014 to 2019 at Peking Union Medical College and Hospital (PUMCH), were retrospectively analyzed as the training set $(n=472)$. Lymph node ultrasound and pathological characteristics were compared using chi-squared and $t$-tests. To verify the accuracy of the model, internal $(n=175)$ and external ( 3 centers, $n=81$ ) validation groups were established for patients in 2019. A nomogram predicting SLN metastasis was constructed using multivariable logistic regression models; discrimination and calibration of the nomogram were assessed by $\mathrm{C}$ statistics and calibration curve. Clinical use by decision curve analysis.

Results: In the training set the $\mathrm{SLN}^{+}$rate was $23.73 \%$. Statistically significant factors associated with SLN positivity were: histology type $(P<0.001)$; $P R(P=0.003)$; Her-2 status $(P=0.049)$; and ultrasound lymph node (ALN-US) shape $(P=0.034)$, corticomedullary demarcation $(P<0.001)$, and blood flow $(P=0.001)$. Five independent variables were integrated into the nomogram (C-statistic 0.714 [ $95 \% \mathrm{Cl}$ : 0.688-0.740], validated internally $(0.816$ [( $95 \% \mathrm{Cl}: 0.784-0.849])$ and externally $(0.942$ [95\% Cl: $0.918-0.966])$. Thus, the model had good predictive accuracy and clinical applicability.

Conclusions: The PUMCH-SLN nomogram provides a direct and reliable tool for prediction and individual evaluation of SLN status preoperatively, emphasizing the role of ALN-ultrasound morphology for predicting SLN metastasis. This nomogram should aid decisions concerning ALN dissection and adjuvant treatment.

\section{Background}

Breast cancer is the most common malignant tumor afflicting women all over the world. In 2018, there were 2.28 million newly diagnosed cases $(1,2)$. Lymph node involvement and tumor size are the most important factors to determine the prognosis of breast cancer, and these remain crucial for strategizing individual treatments (3-5). Historically, axillary lymph node dissection (ALND) has been the reference standard for lymph node (LN) staging. However, for determining metastasis in ALNs, some recent studies found no significant differences in effectiveness between the sentinel lymph node (SLN) procedure and level I and II dissection (6-15). What is more, because of fewer side effects (e.g., paresthesia, lymphedema, and restriction of movement), at many centers SLN dissection/biopsy has replaced ALND as the primary staging procedure $(16,17)$.

For assessing the pathological status of ALNs in patients with stages I-IIIA (T3N1M0) breast cancer, the National Comprehensive Cancer Network (NCCN) (18) recommends SLN biopsy in the surgical staging of clinically negative axilla. Yet, 20 to $60 \%$ of $\mathrm{SLN}^{+}$patients have been found to lack non-SLN (NSLN) involvement after ALND, indicating that these patients received unnecessary axillary treatment (19-22). In a clinical setting with an SLN team of limited experience, risk of false negatives, lack of ultrasound 
standard, and unnecessary axillary treatment, there is increasing practical need to identify SLN status noninvasively, and more precise selection criteria for preoperative SLN biopsy than is offered by the NCCN. Currently, differentiating benign and malignant nodes is aided by mammography, contrastenhanced ultrasound, positron emission tomography-computed tomography, and magnetic resonance imaging (23-25). However, ALN ultrasound (ALN-US) remains the routine tool for assisting SLN evaluation $(3,26)$.

How to use routine examination data to judge the SLN status of patients who have a suspicious ALN at ultrasound, but a negative clinical physical examination, is highly problematic. In this study, ALN-US was evaluated with reference to pathological evidence for differentiating patients with positive or negative SLNs, and a nomogram constructed for predicting SLN metastasis.

\section{Methods}

This study retrospectively analyzed patients who had a suspicious ALN at ultrasound, but a negative clinical physical examination, and were treated with SLN biopsy from 1 January 2014 to 31 December 2019 at the following medical centers: Peking Union Medical College and Hospital (PUMCH); Baoji Maternal and Child Health Hospital; Qinghai Provincial People's Hospital; and Shanxi Traditional Chinese Medical Hospital. Patients were apportioned to a training set of patients at PUMCH from 1 January 2014 to 1 January $2019(n=472)$, an internal validation set, of patients at PUMCH from 1 January 2019 to 31 December $2019(n=175)$, and an external validation set $(n=81)$ of patients treated at 3 centers from 1 January 2019 to 31 December 2019 (described below).

Patients' demographics and clinical features were recorded, including: age; ALN-US findings; postoperative mass histological pathology, size, SLN status; and type of breast surgery. The SLN status, and $\mathrm{SLN}^{+}$rate of the entire population was analyzed. ALN-US and pathological characteristics were compared between patients with positive and negative SLNs.

To select the training set, patients who had undergone SLN dissection $(n=2332)$ were initially reviewed. Potential patients were excluded for the following reasons: combined negative clinical physical examination and negative ultrasound; received preoperative chemotherapy or endocrine therapy; with metastatic breast cancer or other special types of breast cancer; or were lost to follow-up. Altogether, 1826 were excluded, and 472 patients at PUMCH were enrolled in the training set.

For the internal validation set, 175 patients with breast cancer at PUMCH were enrolled. In addition, 81 patients with breast cancer formed the external validation set, from Baoji Maternal and Child Health Hospital, Qinghai Provincial People's Hospital, and Shanxi Traditional Chinese Medical Hospital.

All subjects were women, and provided written informed consent prior to their enrollment for possible contribution to medical research. The complete clinicopathological data of all patients were collected, and all the patients were followed up (Figure 1). 


\section{Ultrasound techniques and characterization of LNs}

ALN-US scans were evaluated by 2 experienced doctors. The size and morphological factors of Level I, II, and III LNs in the axillary and supraclavicular LNs on the side of breast tumors were recorded and evaluated. The morphological criteria included shape, corticomedullary demarcation (CMD), and blood flow on ALN-US. Shape was described as regular or irregular. Asymmetric focal or diffuse cortical thickening $>3 \mathrm{~mm}$ were all classified as unclear ALN-US CMD, and otherwise clear $(27,28)$.

\section{Surgical procedure, pathological evaluation, and adjuvant therapy}

SLN biopsy lymphatic mapping was performed using dual tracer, methylene blue, and indocyanine green (ICG). Surface localization was done by ICG, then we resected the observed blue-stained LNs and enlarged LNs as the SLNs. ICG were used for reconfirming about SLN resected and may have been missed. Every SLN was routinely analyzed via intraoperative frozen sections. Patients with negative SLNs were not given any further axillary treatment. ALN dissection was avoided in most of the patients who met the American College of Surgeons Oncology Group (ACOSOG) Z0011 criteria; otherwise, ALN dissection was performed. All the patients were treated in accordance with the NCCN guidelines (18).

\section{Statistical analysis}

\section{Clinical data}

The clinicopathological and ALN-US data of the patients were analyzed and the patients were followed up. The last date of follow-up was 1 January 2020. The main endpoint of follow-up was death or distant metastasis. The mean follow-up time was 42 months. The chi-squared test $\left(\chi^{2}\right)$ or Fisher's exact test was used to analyze an association between SLN metastasis and clinicopathological features. Comparisons of continuous variables (age, ALN-US size, and Ki67) were performed using Student's $t$-test. The Kaplan Meier model was applied to analyze the survival rate of patients. The statistical analyses were two-sided. $P<0.05$ was considered a statistically significant difference. Statistical analyses were performed using SPSS software (version 24.0; IBM, Chicago, IL) and GraphPad Prism software (version 6.01).

\section{Construction of the nomogram}

Associations between relevant clinicopathological variables plus ALN-US with SLN status were estimated by multivariable logistic regression models, $P$ value $<0.1$ were included, and the odds ratio (OR) and corresponding $95 \%$ confidence interval $(\mathrm{Cl})$ were calculated. The Hosmer-Lemeshow test was used for multivariable analyses, and a forest map to reflect the results of multivariable analysis. Based on the results of the multivariable analysis, independent variables were selected for inclusion in the nomograms to predict SLN metastasis, using statistical software (R 2.14.1, http://www.r-project.org) (16). 
The nomogram was constructed using RMS software. The performance of the model was assessed through discrimination and calibration. Discrimination ability was calculated using $C$ statistics. The $C$ statistic was applied to evaluate the concordance between the observed and predicted outcomes of the model, which is roughly equivalent to the receiver operating characteristic curve. $C$ statistics range from 0.5 and 1 . To evaluate the calibration of the nomogram, a regression smoothing method was used to produce the calibration plots by bootstrapping with 1000 resamples, representing the association between the observed outcome frequencies and the predicted probabilities. The standard curve is a straight line passing through the origin of the coordinate axis with a slope of 1 . The ROC curves were plotted using RROC software.

\section{Clinical use}

Decision curve analysis was performed using the RMDA software and was used to evaluate the clinical value of the nomogram. It was constructed to estimate a net benefit for the prediction model by quantifying the net benefits at different threshold probabilities $(29,30)$.

\section{Results}

\section{Patients clinical, ultrasound, and pathological characteristics}

The training set comprised the data of 472 patients, with 360 and 112, respectively, SLN ${ }^{-}$and SLN $\mathrm{SL}^{+}$. The patients were treated at PUMCH from 1 January 2014 to 1 January 2019. The internal validation cohort consisted of 175 patients, who were treated at PUMCH from 1 January to 31 December 2019. Eighty-one patients made up the external validation cohort. These patients were treated from 1 January to 31 December 2019 at Baoji Maternal and Child Health Hospital, Qinghai Provincial People's Hospital, and Shanxi Traditional Chinese Medical Hospital. The clinical, ultrasound, and pathological characteristics from the multicenter cohorts are listed below.

In the overall training cohort, $23.73 \%$ were $\mathrm{SLN}^{+}$; in the subgroup specifically with ductal carcinoma in situ (DCIS) with micrometastasis, this rate was $11.63 \%$. The median age at diagnosis was 48 years (range, 16$85 \mathrm{y}$ ). The patients' ALN-US and basic clinical pathological characteristics are listed in Table 2 and Figure 2. The patients that were found $S L N^{+}$differed significantly from the $\mathrm{SLN}^{-}$with regard to histology type ( $P$ $<0.001)$; ALN-US shape, CMD, and blood flow $(P=0.034,<0.001, P=0.001$, respectively); PR status ( $P=$ $0.003)$; and Her-2 status $(P=0.049)$. In the subgroup with invasive ductal carcinoma (IDC), the molecular subtypes of the 2 groups were statistically comparable $\left(c^{2}=4.148, P=0.246\right)$.

\section{SLN metastatic prediction model}

Independent variables of $\mathrm{SLN}^{+}$patients

The univariate analysis indicated that histological type, PR, Her-2, ALN-US shape, ALN-US CMD, and ALNUS blood were significantly associated with $\mathrm{SLN}^{+}$status. Based on the univariate analysis, variables with 
a difference of $P<0.1$ were incorporated into the multivariable analysis (Figure $3 \mathrm{~A}$ ). With HosmerLemeshow $\chi 2=4.674, P=0.700>0.05$, this suggests that there is no statistical significance between the predictive value of the model and the actual observed value, and thus the prediction model has a good calibration ability.

\section{Predictive (PUMCH-SLN) nomogram for breast cancer SLN metastasis}

The multivariable logistic regression model found that histological type, PR status, ALN-US shape, CMD, and blood were associated with an increased likelihood of SLN metastasis. IDC histology (odds ratio [OR] 3.20; 95\% Cl 1.77-5.81; $P<0.001$ ); PR positive (OR 2.46; 95\% Cl 1.44-4.23; $P=0.001$ ); ALN-US shape irregular (OR 3.51; 95\% Cl 1.13-10.89; $P=0.030)$; ALN-US CMD unclear (OR 2.81; 95\% Cl 1.60-4.96; $P<$ $0.001)$; and ALN-US blood present (OR 1.98; 95\% Cl 1.24-3.14; $P=0.004)$.

The logistic regression analysis was used to develop a nomogram, which we called the PUMCH-SLN nomogram. The nomogram combined the pathologically-related factors (histological type, PR) and ALNUS morphology (i.e., shape, CMD, and blood; Figure 3A). In the PUMCH-SLN nomogram (Figure 3B), each independent variable corresponds to a specific score when a linear line is draw straight upward to the score axis. The total score refers to the sum of the score of each variable, which reflects the predicted probability of SLN metastasis by drawing a vertical line from the total score axis. Once the total is located, a vertical line is made between the total score and the final row. Row 8 predicts risk of SLN metastasis. For example, for the patient shown in Figure 2 with IDC breast cancer, PR-positive, and ALNUS with regular shape, unclear CMD, and blood (with scores of 100,70 , nil, 80 , and 50 , respectively), the total points equaled a score of 300 , which corresponds to a predicted SLN metastasis rate of $60 \%$.

\section{Calibration and discrimination of the nomogram}

The calibration plots showed acceptable consistency between the prediction via the nomogram and the actual observed outcome, with a mean absolute error $=0.015$ (Figure 4A). The discrimination of the nomograms revealed good prognostic accuracy and clinical applicability, as indicated by the $\mathrm{C}$ statistic 0.714 (95\% Cl: 0.688-0.740; Figure 4B).

\section{Model validation}

To verify further the accuracy of our model, 175 patients with suspected ALN metastasis based on ultrasound were selected from the patients with breast cancer as the internal validation group, treated from 1 January to 31 December 2019 at PUMCH. The external validation group comprised 81 patients treated from 1 January to 31 December 2019 at Baoji Maternal and Child Health Hospital, Qinghai Provincial People's Hospital, and Shanxi Traditional Chinese Medical Hospital.

The c-indexes of the internal and external validation groups were, respectively, 0.816 (95\% Cl: $0.784-$ 0.849 ) and 0.942 (95\% Cl: 0.918-0.966). This further confirmed that the LN prediction model had good predictive accuracy and clinical applicability (Fig. 5 and Fig. 6). 


\section{Clinical use}

The decision curve analysis for the PUMCH-SLN nomogram is shown in Figures $4 \mathrm{C}, 5 \mathrm{C}$, and $6 \mathrm{C}$. The simple model was constructed based on pathological type and PR status, and the complex model was constructed by all variables of the nomogram. Previous research has reported that the rate of LN metastasis is $25 \%$ to $28 \%$ (9). It can be seen that within the threshold range of 0.1 to 0.7 , the net benefit rate of the complex model is higher than that of the simple model. Thus the PUMCH-SLN nomogram showed a high net benefit to inform clinical decisions, and ultrasound can well supplement pathological factors for predicting SLN metastasis.

\section{Survival analysis}

Our data show that patients that are $\mathrm{SLN}^{+}$and $\mathrm{SLN}^{-}$have significantly different rates of disease-free survival (DFS; $P=0.032$; Figure 7), but not overall survival (OS; $P=0.079$ ) (Figure 7). In addition, in the p53 wild-type subgroup, DFS differed significantly between the $\mathrm{SLN}^{+}$and $\mathrm{SLN}^{-}$patients $(P=0.030)$, but this was not observed in the p53 mutant subgroup $(P=0.427)$. In patients who were $\mathrm{SLN}^{+}$(receiving the standard treatment in accordance with the NCCN guidelines), patients with and without ALND had similar DFS and OS (respectively, $P=0.298$ and 0.64 ).

\section{Discussion}

How to use routine examination data to judge SLN status in patients who have a suspicious ALN at ultrasound, but a negative clinical physical examination, is highly problematic. In this study, ALN-US was evaluated relative to pathological characteristics for differentiating patients with positive or negative SLNs, and a nomogram constructed for predicting SLN metastasis. It was found that patients who were $\mathrm{SLN}^{+}$were more likely to demonstrate histological IDC, PR-positive status, and ALN-US with irregular shape, unclear CMD, and the presence of blood flow. We developed a nomogram based on the pathology and ALN-US characteristics to predict SLN metastasis, and it displays excellent ability to predict SLN metastasis, with an area under the $\mathrm{C}$-statistic curve (AUC) of 0.714 . The nomogram can be used to assist clinicians to predict SLN metastasis preoperatively, and thus may assist decisions regarding surgical strategy for patients with breast cancer. It is easy to generalize, simple to use, and more intuitive. The AUC values were verified in validation groups, internally and externally, as 0.816 and 0.942 , respectively. The calibration curve of the nomogram shows that the nomogram prediction is consistent with the actual metastasis rate. Such a nomogram is rare, based as it is on ultrasound and pathological parameters, with complete survival data, and receiving multicenter validation.

Currently, the increasing need for SLN dissection has highlighted concern for a means to predict LN metastasis and aid the selection and treatment of these patients. Scientists have reported many clinical characteristics that are related to LN metastasis, including multifocality, LN palpability, histological type, and $L N$ status determined via magnetic resonance imaging (9,30-32). In addition, Bevilacqua et al. (30) found that patient age, tumor size, grade, and location, lymphovascular invasion, estrogen receptor (ER) 
and PR status are related to LN metastasis (33). Other nomograms to assist LN prediction in breast cancer have been published. For example, Huang et al. (34) constructed a nomogram based on carcinoembryonic antigen (CEA) status, radiomics signature, and LN status depicted on computed tomography. Xie et al.'s (35) nomogram incorporated risk factors (age, tumor size, tumor location, and ALN US-reported status) and miRNA signature. Some others have relied only on a single examination feature, without combining patients' clinical data. The above predictive models, which rely on miRNAs, computed tomography, and magnetic resonance imaging, are not used widely by surgeons, and a nomogram based on ultrasound lacks detailed morphology. Most importantly, a preoperative nomogram hardly exists that was constructed specifically for patients with breast cancer who have a suspicious ALN on US, but a negative clinical physical examination.

In recent years, more and more nomograms have been built using imaging data, for example, predicting ALN in early breast cancer (36), or non-SLN metastasis in patients during neoadjuvant chemotherapy (37). These models share similarities with ours, but are also fundamentally different. The nomogram of the present study has successfully resolved the questions raised above. Its greatest advantage is that LNs can be evaluated preoperatively using only the pathology based on biopsy and ultrasound morphological status. Its practicability and value is not only reflected in its convenience and rapid application, but also avoids false negative results from SLN biopsy to some degree, which internationally ranges from $5.5 \%$ to $43 \%(38)$. What is more, this nomogram can assist decisions to conduct further ALND in some complicated cases of breast cancer.

According to the 2020 NCCN guidelines for breast cancer, further axillary surgery is not recommended for patients with $\mathrm{T} 1$ or $\mathrm{T} 2$ tumor with 1 or 2 positive SLNs, who did not receive preoperative systemic therapy, were treated with lumpectomy, or are prepared to receive whole breast radiation (18). However, there is no consideration of the total number of SLNs resected. For example, in a case in which the intraoperative frozen section of the SLN is $2 / 2$, not 2/4, then the clinician is left with a cofounding decision whether to perform ALN dissection or administer continuous treatment. The present PUMCH-SLN nomogram provides a more detailed reference. Although the Tenon, MSKCC, MDA, Mayo, Cambridge, and Stanford models $(39,40)$ all assess the risk of non-SLN metastasis using SLN and pathology, they are not designed especially for this very situation, and do not incorporate imaging features. The novel feature of our nomogram is that morphological status is incorporated with ALN-US.

In addition to the convenience and easy operation of the present nomogram, to our best knowledge there is no reliable evaluation and reporting standard for ALN, and the overall accuracy of ALN-US remains controversial (35). Because the study of ALNs is subject to strong subjectivity, there is great variability in the judgements made by ultrasound doctors all over the world. In some studies $(27,28)$, LN size, or morphological findings, or both, were used as ultrasound criteria. However, recent studies $(3,26)$ show that the morphological criteria of LNs on ultrasound are more important, with total replacement or eccentric hilus of the $\mathrm{LN}$ and hypo-echoic cortex being the most important morphological ultrasound criteria. Bedi et al. $(19,41)$ emphasized that there is no difference between the size of benign and 
malignant LNs, and cortical morphological findings and hypoechoic cortex are more important than LN size. This is similar to the present results.

Whether the data is qualitative or quantitative, specific values among studies are not consistent. For example, cortical thickness $>5 \mathrm{~mm}$ was identified as the cutoff for metastatic LNs, while diffuse cortical thickening $>3 \mathrm{~mm}$ was reported as suspicious criteria with a specificity of $49 \%$ to $85 \%$, and sensitivity of $96 \%$, in predicting metastatic disease (28). For the present nomogram, diffuse cortical thickening $>3 \mathrm{~mm}$ was considered suspicious criteria, while other morphological indicators are subjective.

We wondered if developing BI-RADS ALN criteria could be applied in breast cancer; such criteria could include detailed evaluation of the eccentric placement, complete anechoic or hypoechoic appearance of the LN, axillary and echogenic hilus obliteration, asymmetric cortical thickening, and other possible quantitative evaluations gained from ultrasound ALN-US. Meanwhile, our team is developing another artificial intelligence-assisted SLN prediction system. In this way metastatic LNs could be identified by ultrasonography with high sensitivity and positive predictive value, assisting in the stratification of patients with ALN metastasis, aiding in therapy planning and patient staging, and ultimately contributing to improvements in surgery strategy and survival rates.

T-staging must be discussed. First, the pathological T stage cannot be gained before surgery; only the tumor size may be determined by ultrasound. In the research of Fidan et al. (28), histopathological tumor size correlated with the primary tumor size measured. In the present study, we were not able to differentiate SLN positivity by the tumor size shown on ultrasound (Table 1). Here, it should be noted that although some DCIS are large in diameter, they have a lower risk of metastasis. Meanwhile, a subgroup analysis of patients with IDC was conducted in this study, using the chi-squared and Fisher's exact tests. The results showed that, with $\chi 2=6.333$ and $P=0.032$, there was a statistical difference. Yet, about $25 \%$ of women with seemingly pure DCIS on initial biopsy will be found with invasive breast cancer at the time of the definitive surgical procedure (42), and thus will ultimately require ALN staging (18). In any case, primary tumor size is an important factor affecting ALN metastasis $(4,5)$. In the present study the pathological T stages of all patients were compared, and there were statistical differences $(X 2 \rrbracket 22.067, P<$ 0.001). However, statistics for the last two $T$ stages are obtained at the postoperative detailed evaluation of pathological results, and therefore cannot be used as a reference before surgery at this time. Therefore, we can acknowledge that pathological T staging is related to $L N$ metastasis in all patients with breast cancer, but the T parameter was not included in the nomogram for preoperative use developed in this study.

In the survival analysis, the DFS of the $\mathrm{SLN}^{+}$patients was significantly shorter than that of the $\mathrm{SNL}^{-}$ patients, and a similar trend has been reported in key clinical trials (43). The two groups are similar in overall survival, perhaps because of short follow-up time, and SLN dissection is performed in patients with relatively early stage breast cancer. In addition, in the present study p53 expression was associated with prognosis. This poses questions that warrant more basic research and large-scale clinical trials in all patients with breast cancer. 


\section{Conclusion}

One in four patients with a suspicious ALN on ultrasound, but a negative clinical physical examination, had ALN metastasis. The PUMCH-SLN nomogram constructed in this study shows that patients with IDC, PR-positive status, and ALN-US with irregular shape, unclear CMD, and presence of blood flow are more likely to be SLN ${ }^{+}$. The PUMCH-SLN nomogram should be of great benefit to inform clinical decisions. Ultrasound morphological characteristics are a highly credible reference to supplement pathology for predicting SLN metastasis. The nomogram is a convenient practical tool to warn preoperatively of SLN false-negative patients. This nomogram can also assist in decisions about further ALND in some patients with complicated breast cancer.

\section{Abbreviations}

ALN, axillary lymph node; $\mathrm{Cl}$, confidence interval; CMD, corticomedullary demarcation; DCIS, ductal carcinoma in situ, DFS, disease-free survival; ER, estrogen receptor; ICG, indocyanine green; IDC, invasive ductal carcinoma; SLN, sentinel lymph node; OR, odds ratio; OS, overall survival; PR, progesterone receptor; PUMCH, Peking Union Medical College and Hospital; US, ultrasound;

\section{Declarations}

\section{Ethics approval and consent to participate}

All procedures performed in studies involving human participants were in accordance with the ethical standards of the institutional and/or national research committee and with the 1964 Helsinki declaration and its later amendments or comparable ethical standards. Written informed consent was obtained from all individual participants included in the study.

\section{Consent for Publication}

All data published here are under the consent for publication. Written informed consent was obtained from all individual participants included in the study.

\section{Availability of data and materials}

The datasets generated and analyzed during the present study are available from the corresponding author on reasonable request.

\section{Conflict of Interest}

Each author declared that he/she has no conflict of interest

\section{Funding}


This work was supported by Tsinghua University-Peking Union Medical College Hospital Initiative Scientific Research Program, No. $2019 Z$.

\section{Authors' contributions}

Conception and design, Writing, review and/or revision of the manuscript: W-XF

Development of methodology: Z-GC

Acquisition of data: Z-QL, L-ZZ, W-SF

Analysis andinterpretation of data: Z-ZCQY-CL, M-XY, SY, SH

Administrative, technical, or material support: SQ, Z-YD, M-F, L-Y, S-SJ, Z-XH, Z-Y

Study supervision: L-JX; D-JH

\section{Acknowledgements}

Not applicable

\section{References}

1. Bray F, Ferlay J, Soerjomataram I, Siegel RL, Torre LA, Jemal A. Global cancer statistics 2018: GLOBOCAN estimates of incidence and mortality worldwide for 36 cancers in 185 countries. CA Cancer J Clin 2018; 68: 394-424.

2. Chen W, Zheng R, Baade PD, Zhang S, Zeng H, Bray F, et al. Cancer statistics in China, 2015. CA Cancer J Clin 2016; 66: 115-132.

3. Alvarez S, Añorbe E, Alcorta P, López F, Alonso I, Cortés J. Role of sonography in the diagnosis of axillary lymph node metastases in breast cancer: a systematic review. AJR Am J Roentgenol 2006; 186: 1342-1348.

4. García Fernández A, Fraile M, Giménez N, Reñe A, Torras M, Canales L, et al. Use of axillary ultrasound, ultrasound-fine needle aspiration biopsy and magnetic resonance imaging in the preoperative triage of breast cancer patients considered for sentinel node biopsy. Ultrasound Med Biol 2011; 37: 16-22.

5. Koelliker SL, Chung MA, Mainiero MB, Steinhoff MM, Cady B. Axillary lymph nodes: US-guided fineneedle aspiration for initial staging of breast cancer-correlation with primary tumor size. Radiology 2008; 246: 81-89.

6. Bass SS, Lyman GH, McCann CR, Ku NN, Berman C, Durand K, et al. Lymphatic Mapping and Sentinel Lymph Node Biopsy. Breast J 1999; 5: 288-295.

7. Cox CE. Lymphatic mapping in breast cancer: combination technique. Ann Surg Oncol 2001; 8: 67s70 s. 
8. Cox CE, Nguyen K, Gray RJ, Salud C, Ku NN, Dupont E, et al. Importance of lymphatic mapping in ductal carcinoma in situ (DCIS): why map DCIS? Am Surg 2001; 67: 513-519; discussion 519-521.

9. Krag D, Weaver D, Ashikaga T, Moffat F, Klimberg VS, Shriver C, et al. The sentinel node in breast cancer--a multicenter validation study. N Engl J Med 1998; 339: 941-946.

10. Krag DN, Anderson SJ, Julian TB, Brown AM, Harlow SP, Costantino JP, et al. Sentinel-lymph-node resection compared with conventional axillary-lymph-node dissection in clinically node-negative patients with breast cancer: overall survival findings from the NSABP B-32 randomised phase 3 trial. Lancet Oncol 2010; 11: 927-933.

11. Kuehn T, Vogl FD, Helms G, Pueckler SV, Schirrmeister H, Strueber R, et al. Sentinel-node biopsy for axillary staging in breast cancer: results from a large prospective German multi-institutional trial. Eur J Surg Oncol 2004; 30: 252-259.

12. McMasters KM, Giuliano AE, Ross MI, Reintgen DS, Hunt KK, Byrd DR, et al. Sentinel-lymph-node biopsy for breast cancer-not yet the standard of care. N Engl J Med 1998; 339: 990-995.

13. O'Hea BJ, Hill AD, El-Shirbiny AM, Yeh SD, Rosen PP, Coit DG, et al. Sentinel lymph node biopsy in breast cancer: initial experience at Memorial Sloan-Kettering Cancer Center. J Am Coll Surg 1998; 186: $423-427$.

14. Veronesi U, Paganelli G, Viale G, Luini A, Zurrida S, Galimberti V, et al. A randomized comparison of sentinel-node biopsy with routine axillary dissection in breast cancer. N Engl J Med 2003; 349: 546553.

15. Mansel RE, Fallowfield L, Kissin M, Goyal A, Newcombe RG, Dixon JM, et al. Randomized multicenter trial of sentinel node biopsy versus standard axillary treatment in operable breast cancer: the ALMANAC Trial. J Natl Cancer Inst 2006; 98: 599-609.

16. Purushotham AD, Upponi S, Klevesath MB, Bobrow L, Millar K, Myles JP, et al. Morbidity after sentinel lymph node biopsy in primary breast cancer: results from a randomized controlled trial. J Clin Oncol 2005; 23: 4312-4321.

17. Gentilini O, Veronesi U. Abandoning sentinel lymph node biopsy in early breast cancer? A new trial in progress at the European Institute of Oncology of Milan (SOUND: Sentinel node vs Observation after axillary UltraSouND). Breast 2012; 21: 678-681.

18. Weber M, Bellwald D, Wingenfeld C, Hempfing A, Leunig M. The avascular talus: revascularization in an animal model. Foot Ankle Int 2004; 25: 151-158.

19. Chen K, Zhu L, Jia W, Rao N, Fan M, Huang H, et al. Validation and comparison of models to predict non-sentinel lymph node metastasis in breast cancer patients. Cancer Sci 2012; 103: 274-281.

20. Pal A, Provenzano E, Duffy SW, Pinder SE, Purushotham AD. A model for predicting non-sentinel lymph node metastatic disease when the sentinel lymph node is positive. Br J Surg 2008; 95: 302309.

21. Chagpar AB, Scoggins CR, Martin RC, 2nd, Carlson DJ, Laidley AL, El-Eid SE, et al. Prediction of sentinel lymph node-only disease in women with invasive breast cancer. Am J Surg 2006; 192: 882887. 
22. Van Zee KJ, Manasseh DM, Bevilacqua JL, Boolbol SK, Fey JV, Tan LK, et al. A nomogram for predicting the likelihood of additional nodal metastases in breast cancer patients with a positive sentinel node biopsy. Ann Surg Oncol 2003; 10: 1140-1151.

23. Peare R, Staff RT, Heys SD. The use of FDG-PET in assessing axillary lymph node status in breast cancer: a systematic review and meta-analysis of the literature. Breast Cancer Res Treat 2010; 123 : 281-290.

24. Mortellaro VE, Marshall J, Singer L, Hochwald SN, Chang M, Copeland EM, et al. Magnetic resonance imaging for axillary staging in patients with breast cancer. J Magn Reson Imaging 2009; 30: $309-312$.

25. Piscaglia F, Nolsøe C, Dietrich CF, Cosgrove DO, Gilja OH, Bachmann Nielsen M, et al. The EFSUMB Guidelines and Recommendations on the Clinical Practice of Contrast Enhanced Ultrasound (CEUS): update 2011 on non-hepatic applications. Ultraschall Med 2012; 33: 33-59.

26. Houssami N, Ciatto S, Turner RM, Cody HS, 3rd, Macaskill P. Preoperative ultrasound-guided needle biopsy of axillary nodes in invasive breast cancer: meta-analysis of its accuracy and utility in staging the axilla. Ann Surg 2011; 254: 243-251.

27. Farrokh D, Ameri L, Oliaee F, Maftouh M, Sadeghi M, Forghani MN, et al. Can ultrasound be considered as a potential alternative for sentinel lymph node biopsy for axillary lymph node metastasis detection in breast cancer patients? Breast J 2019; 25: 1300-1302.

28. Fidan N, Ozturk E, Yucesoy C, Hekimoglu B. Preoperative Evaluation of Axillary Lymph Nodes in Malignant Breast Lesions with Ultrasonography and Histopathologic Correlation. J Belg Soc Radiol 2016; 100: 58.

29. Vickers AJ, Cronin AM, Elkin EB, Gonen M. Extensions to decision curve analysis, a novel method for evaluating diagnostic tests, prediction models and molecular markers. BMC Med Inform Decis Mak 2008; 8: 53.

30. Bevilacqua JL, Kattan MW, Fey JV, Cody HS, 3rd, Borgen PI, Van Zee KJ. Doctor, what are my chances of having a positive sentinel node? A validated nomogram for risk estimation. J Clin Oncol 2007; 25: 3670-3679.

31. Viale G, Zurrida S, Maiorano E, Mazzarol G, Pruneri G, Paganelli G, et al. Predicting the status of axillary sentinel lymph nodes in 4351 patients with invasive breast carcinoma treated in a single institution. Cancer 2005; 103: 492-500.

32. Wu JL, Tseng HS, Yang LH, Wu HK, Kuo SJ, Chen ST, et al. Prediction of axillary lymph node metastases in breast cancer patients based on pathologic information of the primary tumor. Med Sci Monit 2014; 20: 577-581.

33. Han L, Zhu Y, Liu Z, Yu T, He C, Jiang W, et al. Radiomic nomogram for prediction of axillary lymph node metastasis in breast cancer. Eur Radiol 2019; 29: 3820-3829.

34. Huang YQ, Liang CH, He L, Tian J, Liang CS, Chen X, et al. Development and Validation of a Radiomics Nomogram for Preoperative Prediction of Lymph Node Metastasis in Colorectal Cancer. J Clin Oncol 2016; 34: 2157-2164. 
35. Xie X, Tan W, Chen B, Huang X, Peng C, Yan S, et al. Preoperative prediction nomogram based on primary tumor miRNAs signature and clinical-related features for axillary lymph node metastasis in early-stage invasive breast cancer. Int J Cancer 2018; 142: 1901-1910.

36. Zong Q, Deng J, Ge W, Chen J, Xu D. Establishment of Simple Nomograms for Predicting Axillary Lymph Node Involvement in Early Breast Cancer. Cancer Manag Res 2020; 12: 2025-2035.

37. Cheng $M$, Zhuang $X$, Zhang L, Zhu T, Lin $Y$, Yang $M$, et al. A nomogram to predict non-sentinel lymph node metastasis in patients with initial $\mathrm{cN}+$ breast cancer that downstages to $\mathrm{cNO}$ after neoadjuvant chemotherapy. J Surg Oncol 2020.

38. Wong J, Yong WS, Thike AA, Iqbal J, Salahuddin AS, Ho GH, et al. False negative rate for intraoperative sentinel lymph node frozen section in patients with breast cancer: a retrospective analysis of patients in a single Asian institution. J Clin Pathol 2015; 68: 536-540.

39. Zhu L, Jin L, Li S, Chen K, Jia W, Shan Q, et al. Which nomogram is best for predicting non-sentinel lymph node metastasis in breast cancer patients? A meta-analysis. Breast Cancer Res Treat 2013; 137: 783-795.

40. Wang NN, Yang ZJ, Wang X, Chen LX, Zhao HM, Cao WF, et al. A mathematical prediction model incorporating molecular subtype for risk of non-sentinel lymph node metastasis in sentinel lymph node-positive breast cancer patients: a retrospective analysis and nomogram development. Breast Cancer 2018; 25: 629-638.

41. Bedi DG, Krishnamurthy R, Krishnamurthy S, Edeiken BS, Le-Petross H, Fornage BD, et al. Cortical morphologic features of axillary lymph nodes as a predictor of metastasis in breast cancer: in vitro sonographic study. AJR Am J Roentgenol 2008; 191: 646-652.

42. Brennan ME, Turner RM, Ciatto S, Marinovich ML, French JR, Macaskill P, et al. Ductal carcinoma in situ at core-needle biopsy: meta-analysis of underestimation and predictors of invasive breast cancer. Radiology 2011; 260: 119-128.

43. Giuliano AE, McCall L, Beitsch P, Whitworth PW, Blumencranz P, Leitch AM, et al. Locoregional recurrence after sentinel lymph node dissection with or without axillary dissection in patients with sentinel lymph node metastases: the American College of Surgeons Oncology Group Z0011 randomized trial. Ann Surg 2010; 252: 426-432; discussion 432-423.

\section{Tables}

Table 1. Clinical characteristics of enrolled patients from the multicenter cohorts * 


\begin{tabular}{|c|c|c|c|c|}
\hline & & Training & $\begin{array}{l}\text { Validation } \\
\text { Internal }\end{array}$ & Integrated external \\
\hline Subjects, $\mathrm{n}$ & & 472 & 175 & 81 \\
\hline Age, $y$ & & $49.61 \pm 11.59$ & $50.82 \pm 11.59$ & $51.43 \pm 9.56$ \\
\hline ALN-US Size, cm & & $1.49 \pm 0.62$ & $1.53 \pm 0.61$ & $1.45 \pm 0.63$ \\
\hline \multirow[t]{2}{*}{ ALN-US Shape } & Regular & $457(96.8)$ & $125(71.4)$ & $48(59.3)$ \\
\hline & Irregular & $15(3.2)$ & $50(28.6)$ & $33(40.7)$ \\
\hline \multirow[t]{2}{*}{ ALN-US CMD } & Clear & $400(84.7)$ & $140(80.0)$ & $48(59.3)$ \\
\hline & Unclear & $72(15.3)$ & $35(20.0)$ & $33(40.7)$ \\
\hline \multirow[t]{2}{*}{ ALN-US Blood } & Absent & $244(51.7)$ & $67(38.3)$ & $42(51.9)$ \\
\hline & Present & $238(48.3)$ & $108(61.7)$ & $39(48.1)$ \\
\hline \multirow[t]{2}{*}{ Histological types } & DCIS & $129(27.3)$ & $21(12.0)$ & $6(7.41)$ \\
\hline & IDC & $343(72.7)$ & $154(88.0)$ & $75(92.59)$ \\
\hline \multirow[t]{3}{*}{ Tumor size, cm } & $\leq 2$ & $302(64.0)$ & $75(42.9)$ & $11(13.6)$ \\
\hline & $2-5$ & $159(33.7)$ & $99(56.6)$ & $62(76.5)$ \\
\hline & $>5$ & $11(2.3)$ & $1(0.6)$ & $8(9.9)$ \\
\hline \multirow[t]{2}{*}{ ER } & Positive & $354(75.0)$ & $137(78.3)$ & $51(63.0)$ \\
\hline & Negative & $118(25.0)$ & $38(21.7)$ & $30(37.0)$ \\
\hline \multirow[t]{2}{*}{ PR } & Positive & $322(68.2)$ & $120(68.6)$ & $46(56.8)$ \\
\hline & Negative & $150(31.8)$ & $55(31.4)$ & $35(43.2)$ \\
\hline \multirow[t]{2}{*}{ Her-2 } & Positive & $122(25.8)$ & $39(22.3)$ & $5(6.2)$ \\
\hline & Negative & $350(74.2)$ & $136(77.7)$ & $76(93.8)$ \\
\hline
\end{tabular}

* Reported as n (\%), unless noted otherwise.

Table 2. ALN-US and basic clinical pathological characteristics of the SLN ${ }^{-}$and $\mathrm{SLN}^{+}$patients in the training cohort

\begin{tabular}{|c|c|c|c|c|}
\hline & & SLN (-) & SLN (+) & $P$ \\
\hline Subjects, $\mathrm{n}$ & & 360 & 112 & \\
\hline Age, $y$ & & $50.12 \pm 11.74$ & $47.97 \pm 10.98$ & 0.087 \\
\hline ALN-US Size, $\mathrm{cm}$ & & $1.50 \pm 0.62$ & $1.44 \pm 0.63$ & 0.359 \\
\hline \multirow{2}{*}{ ALN-US Shape } & Regular & $352(97.8)$ & $105(93.8)$ & 0.034 \\
\hline & Irregular & $8(2.2)$ & $7(6.3)$ & \\
\hline \multirow[t]{2}{*}{ ALN-US CMD } & Clear & $319(88.6)$ & $81(72.3)$ & $<0.001$ \\
\hline & Unclear & $41(11.4)$ & $31(27.7)$ & \\
\hline \multirow[t]{2}{*}{ ALN-US blood } & Absent & $201(55.8)$ & $43(38.4)$ & 0.001 \\
\hline & Present & $159(44.2)$ & 69 (61.6) & \\
\hline \multirow[t]{2}{*}{ Histological types } & DCIS & $114(33.6)$ & $15(16.1)$ & $<0.001$ \\
\hline & IDC & $246(66.4)$ & 97 (83.9) & \\
\hline \multirow[t]{3}{*}{ Tumor size } & T1 & $226(62.8)$ & $67.9(16.8)$ & 0.274 \\
\hline & $\mathrm{T} 2$ & $127(35.3)$ & $28.6(50.5)$ & \\
\hline & T3 & $7(1.9)$ & $3.6(6.3)$ & \\
\hline \multirow[t]{2}{*}{ ER } & Positive & $263(73.1)$ & $91(81.3)$ & 0.080 \\
\hline & Negative & $97(26.9)$ & $21(18.8)$ & \\
\hline \multirow[t]{2}{*}{$\mathrm{PR}$} & Positive & $233(64.7)$ & $89(79.5)$ & 0.003 \\
\hline & Negative & $127(35.3)$ & $23(20.5)$ & \\
\hline \multirow[t]{2}{*}{ Her-2 } & Positive & $101(28.1)$ & $21(18.8)$ & 0.049 \\
\hline & Negative & $259(71.9)$ & $91(81.3)$ & \\
\hline \multicolumn{2}{|l|}{$\mathrm{Ki} 67, \%$} & $29.86 \pm 23.49$ & $29.96 \pm 21.63$ & 0.968 \\
\hline
\end{tabular}

\section{Figures}



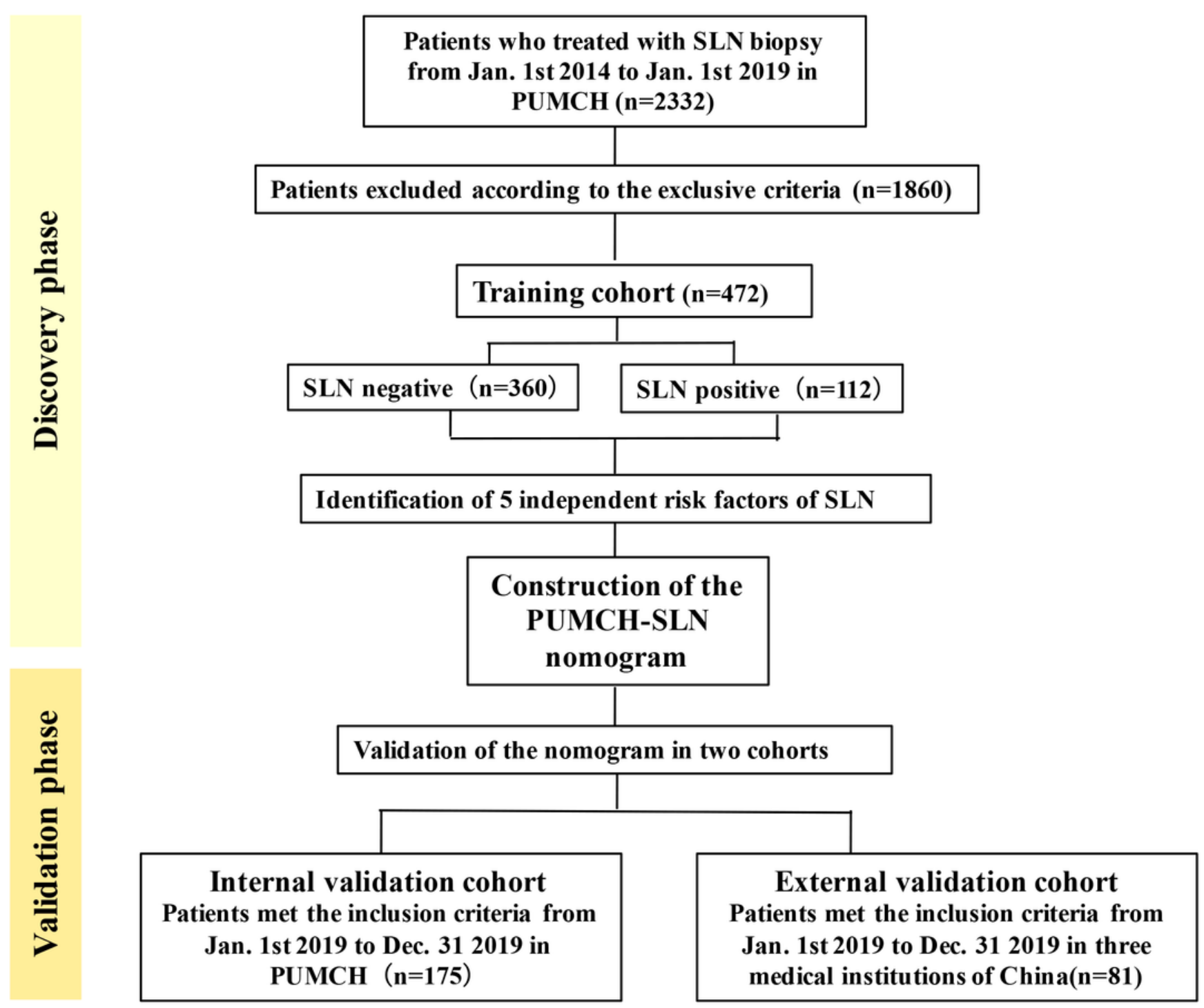

Figure 1

Flow chart of patient screening and exclusion criteria. 

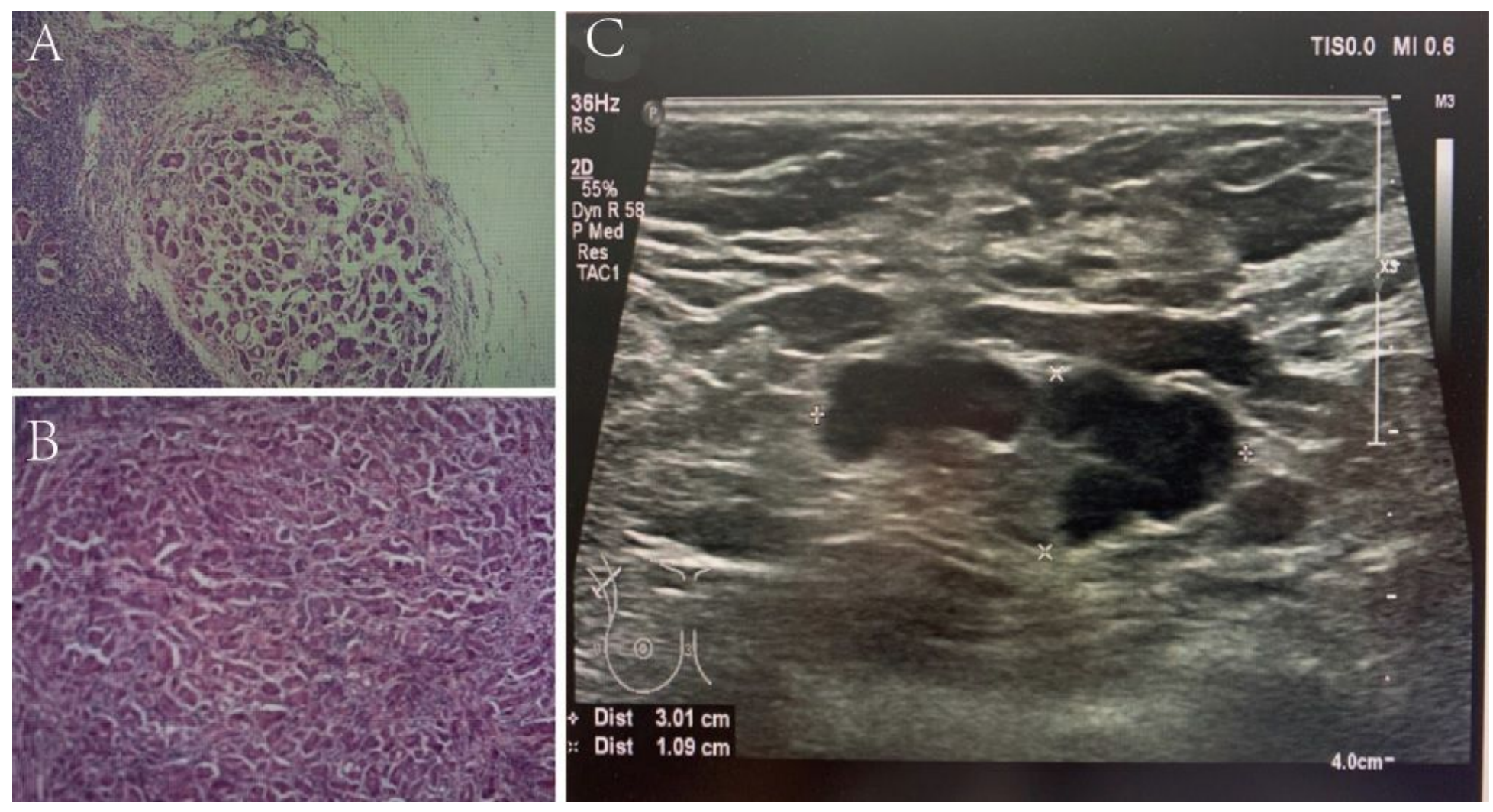

Figure 2

ALN-US and pathology of representative patient with LN metastatic breast cancer. Patient is 43-year-old women with breast cancer: IDC, T1N3M0. Immunohistochemistry: ER+, PR+, Her-2+++, and Ki67 60\%. (A, B) US, pathology; (C) US, LN characterized by 3-cm size, regular shape, unclear CMD, and blood flow.

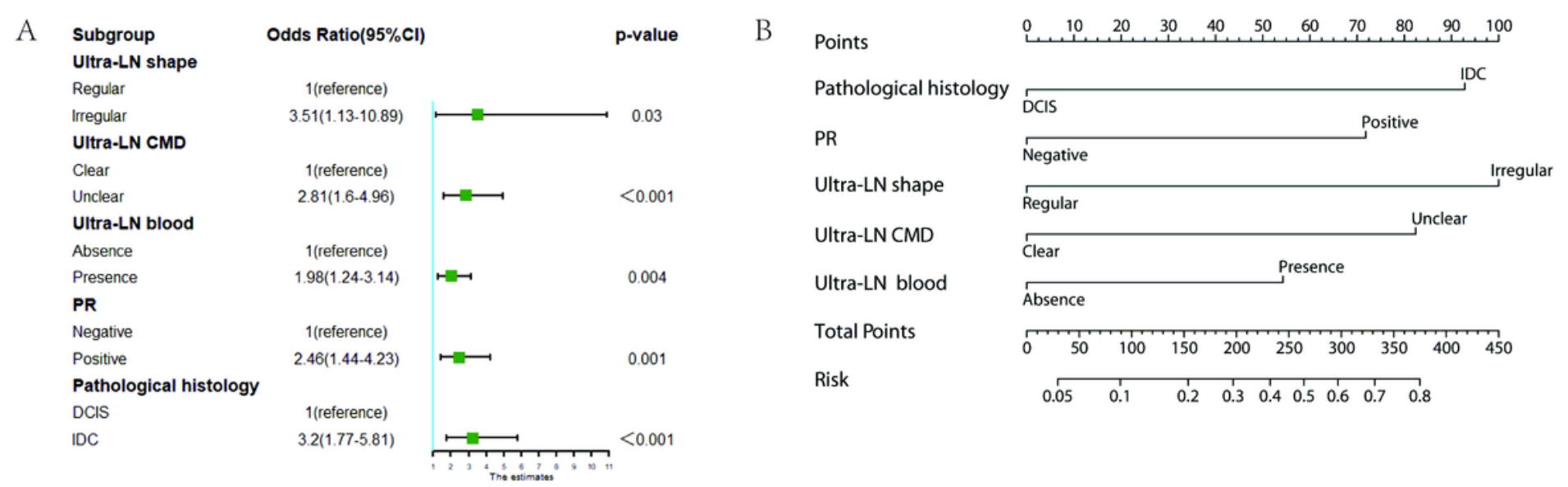

\section{Figure 3}

PUMCH-SLN nomogram and risk factors. (A) Forest map of the multivariable analysis. (B) PUMCH-SLN nomogram for the prediction of LN metastasis 


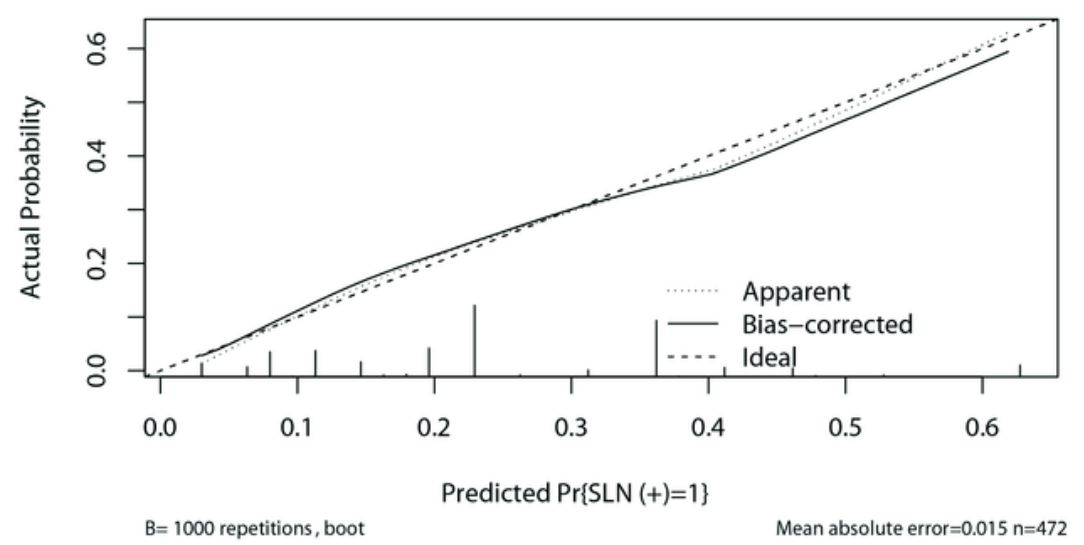

B

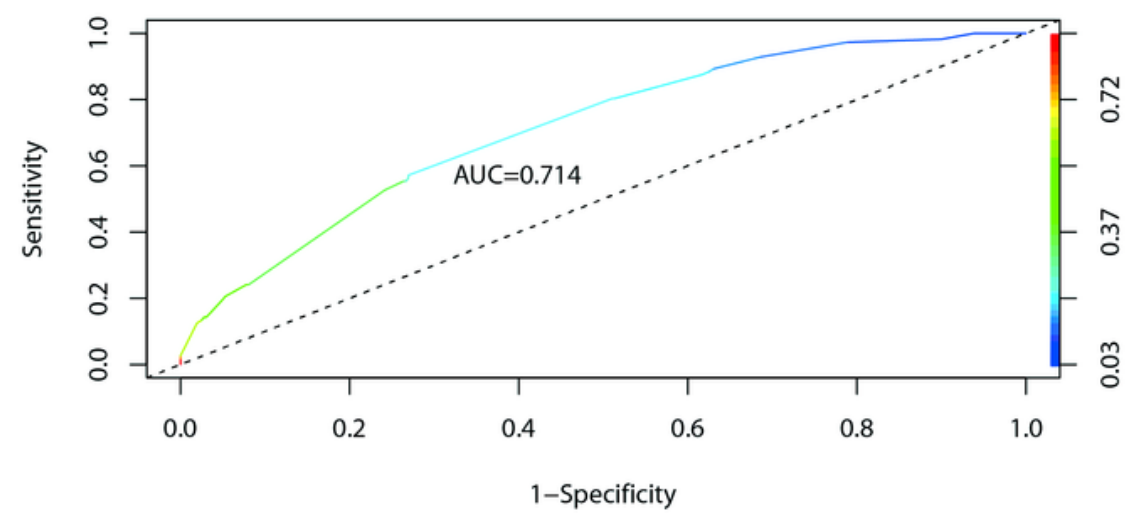

C

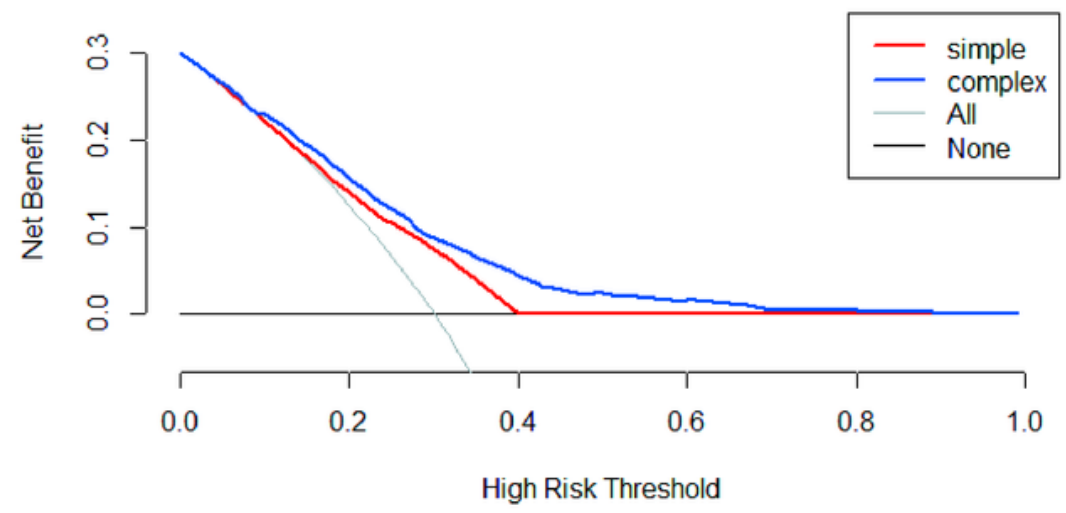

\section{Figure 4}

Calibration, discrimination, and clinical use of the nomogram in the training set. (A) Calibration curves of the PUMCH-SLN nomogram. Calibration curves depict the calibration of the nomogram in terms of agreement between the predicted risk of SLN metastasis and actual SLN metastasis. The $45^{\circ}$ oblique line represents a perfect prediction, and the dotted lines represent the predictive performance of the nomogram. The closer the dotted line fits to the ideal line, the better the predictive accuracy of the 
nomogram. After 1000 resampling, the predicted value of the model fits well with the actual observed value, which is near the $45^{\circ}$ oblique line: mean absolute error 0.015 ; degree of discrimination C-statistic 0.714 (95\% Cl: 0.688-0.740). (B) Plot showing the receiver operating characteristic curve of the PUMCHSLN nomogram. (C) Decision curve analysis for the PUMCH-SLN nomogram. The y-axis represents the net benefit. The blue line represents the PUMCH-SLN nomogram. The red line represents the only use of pathological variables (histological types and PR status) to predict SLN metastasis. The black line represents the hypothesis that no patients had LN metastasis. The $x$-axis represents the threshold probability. The decision curves showed that if the threshold probability is between 0.1 and 0.7 , then using the PUMCH-SLN nomogram to predict SLN metastasis adds more benefit than using only pathological factors 

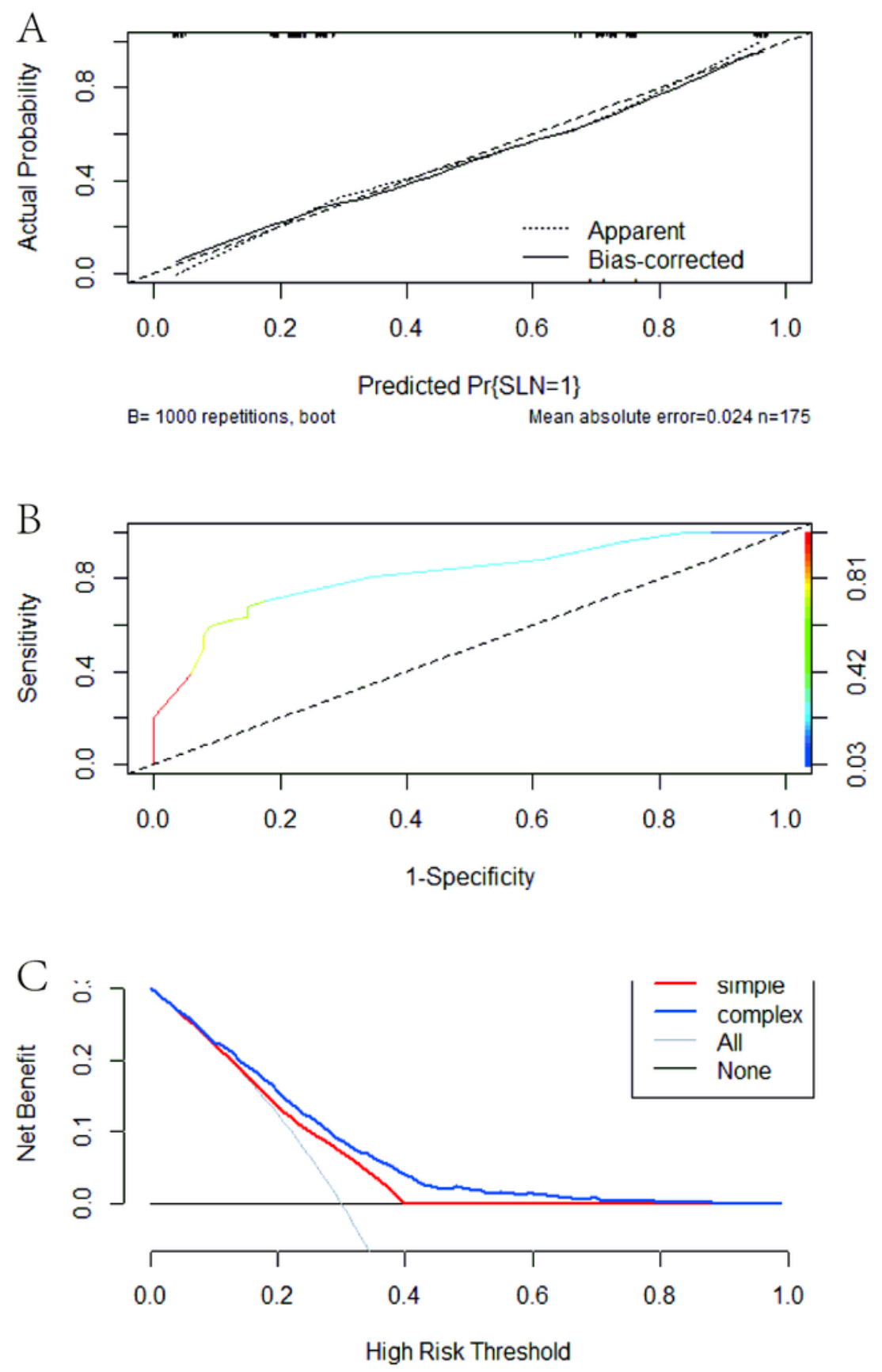

Figure 5

Calibration, discrimination, and clinical use of the nomogram in internal validation set. (A) Calibration curve of the PUMCH-SLN nomogram internal validation group. C index 0.816 (95\% Cl: 0.784-0.849). (B) The receiver operating characteristic curve of the PUMCH-SLN nomogram internal validation group. (C) The decision curve analysis of the PUMCH-SLN nomogram internal validation group. 

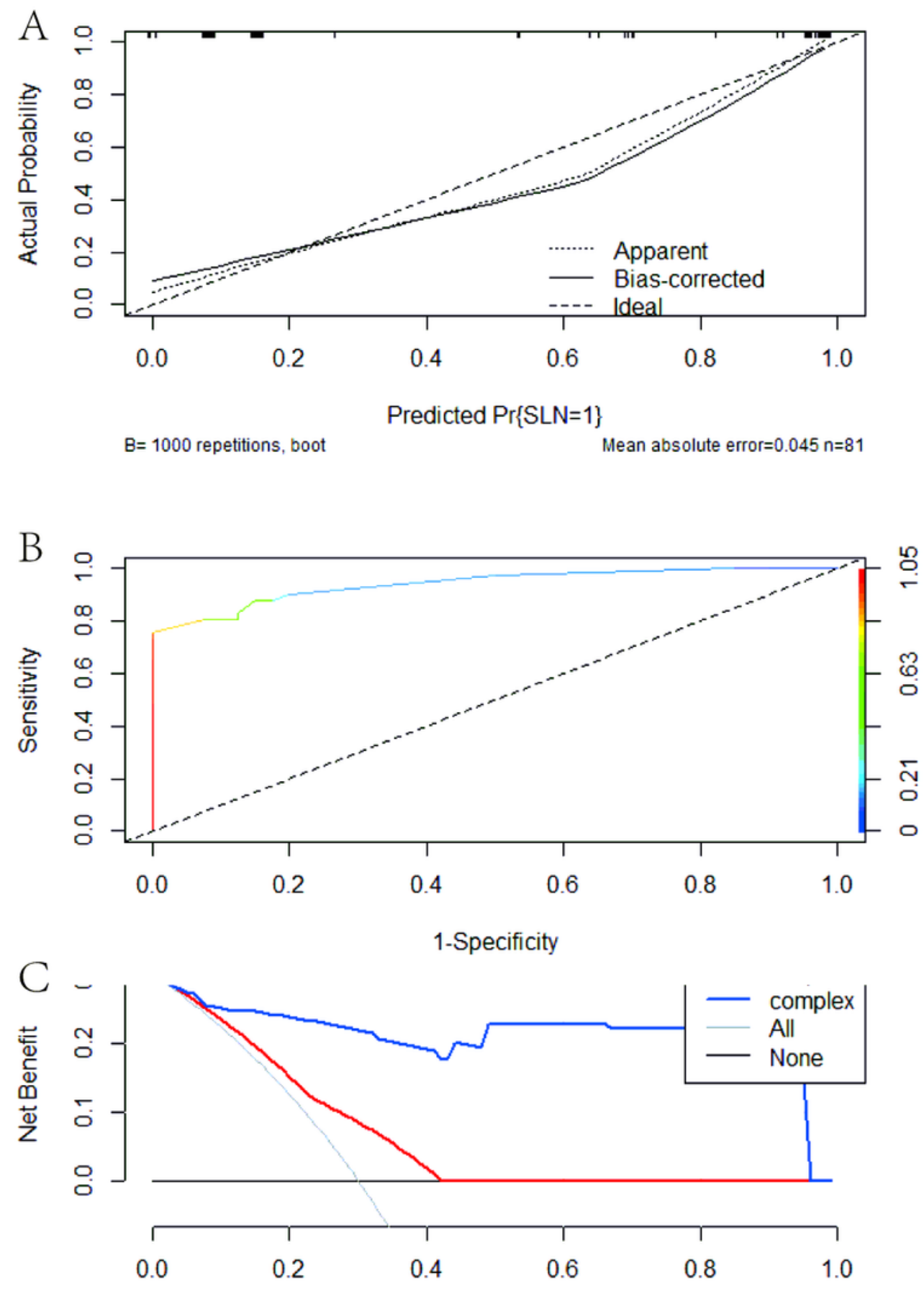

High Risk Threshold

\section{Figure 6}

Calibration, discrimination, and clinical use of the nomogram in external validation set. (A) Calibration curve of the PUMCH-SLN nomogram external validation group. C index 0.942 (95\% Cl: 0.918-0.966). (B) The receiver operating characteristic curve of the PUMCH-SLN nomogram external validation group. (C) Decision curve analysis of the PUMCH-SLN nomogram external validation group. 

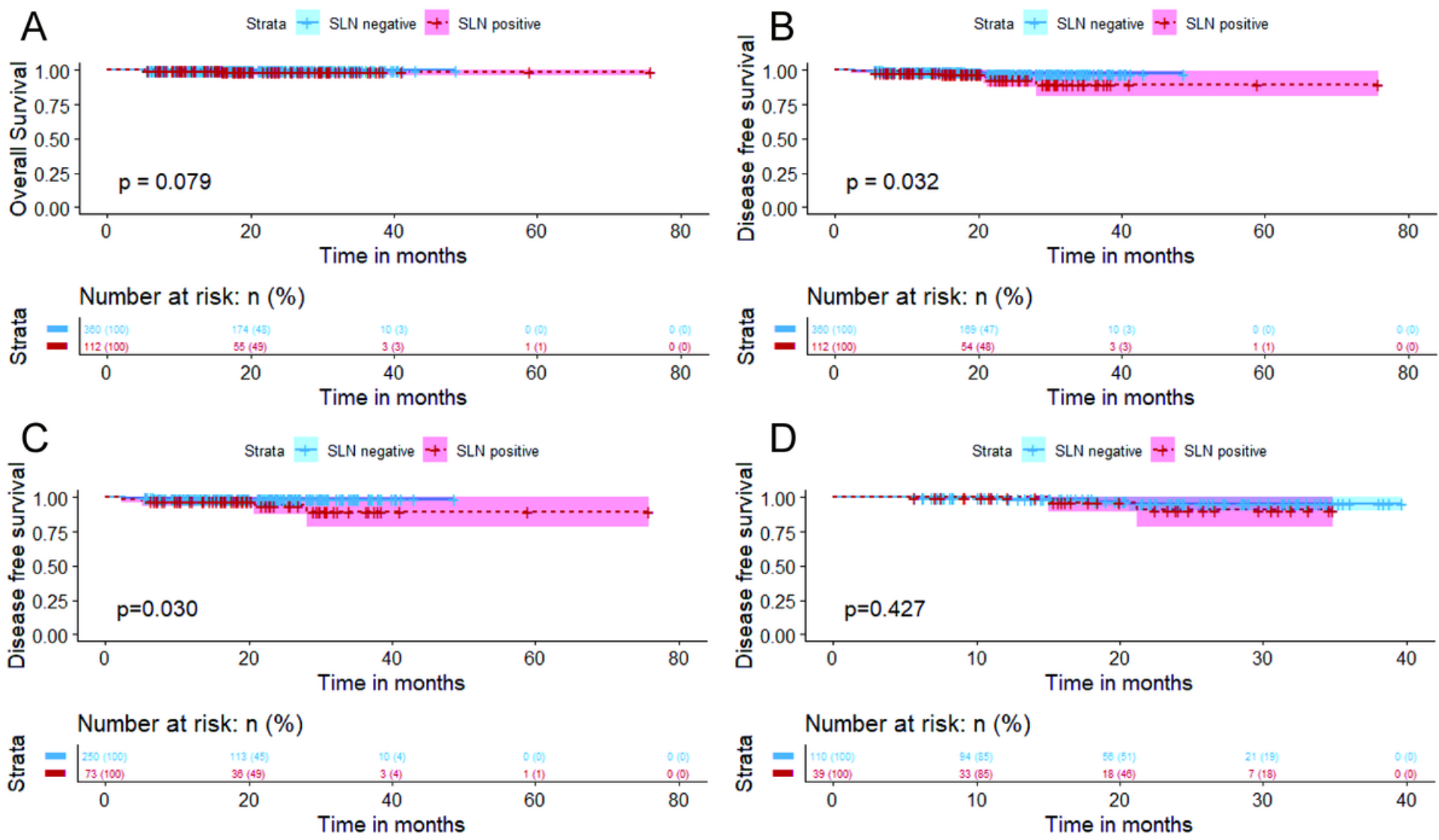

Figure 7

Survival analysis of the SLN+ and SLN- groups. (A) There was no significant difference in OS between the SLN+ group and the SLN- group $(P=0.079)$. (B) There was a significant difference in DFS between the SLN+ group and the SLN- group $(P=0.032)$. (C) There was a significant difference in DFS between the SLN+ and the SLN- patients in the p53 wild type subgroup $(P=0.030)$. (D) There was a significant difference in DFS between the SLN+ group and SLN- group $(P=0.079)$. There was no significant difference in DFS between the SLN+ and the SLN- group patients in the p53 mutant subgroup $(P=$ 0.427). 